\title{
A portable and low-cost fMRI compatible pneumatic system for the investigation of the somatosensensory system in clinical and research environments
}

\author{
Christian Wienbruch ${ }^{\mathrm{c}, 1}$, Victor Candia ${ }^{\mathrm{a}, \mathrm{b}, *, 1}$, Jonas Svensson ${ }^{\mathrm{a}}$, \\ Raimund Kleiser ${ }^{\mathrm{a}}$, Spyros S. Kollias ${ }^{\mathrm{a}}$ \\ ${ }^{a}$ Institute of Neuroradiology, University Hospital of Zurich, Frauenklinikstrasse 10, CH-8091 Zurich, Switzerland \\ ${ }^{\mathrm{b}}$ Collegium Helveticum, University of Zurich and ETH Zurich, Schmelzbergstrasse 25, CH-8092 Zurich, Switzerland \\ ${ }^{\mathrm{c}}$ Department of Psychology, University of Konstanz, Fach D25, D-78457 Konstanz, Germany
}

\begin{abstract}
There still is a need for devices that allow reproducible stimulation of skin areas of the human body. We constructed a stimulation system and tested it by using brief pneumatic stimulation to the right thumb of nine healthy volunteers. BOLD-signals in response to tactile stimulation with frequencies of 1,3 and $5 \mathrm{~Hz}$ were measured using a 3 T MRI scanner. The stimulation device consists of synthetic membranes connected to plastic tubes capable of carrying compressed air, and an electronic component, which controls the on- and off-switching of an electromagnetic valve. The valve near the MR-scanner did not lower the image quality. Primary somatosensory activation contralateral to the stimulation site was reliably detected in response to a stimulus magnitude of 3.5 bar in all volunteers. $1 \mathrm{~Hz}$ stimulation resulted in higher maximal percentage BOLD-signal changes. Our device is an easy-to-construct, low-cost and portable tool suitable for research and clinical environments. It permits passive non-painful stimulation relevant for clinical assessments and is also compatible with magnetoencephalography (MEG) and electroencephalography (EEG). In basic and clinical research, this device therefore contributes to meaningful comparisons between results obtained with different techniques.
\end{abstract}

Keywords: MRI compatible devices; Pneumatic stimulation; fMRI; Sensory system

Magnetoencephalography (MEG), electroencephalography (EEG) and functional magnetic resonance imaging (fMRI), impose limitations on the applicability of stimulation devices used to induce peripheral stimulation. Such limitations include, for example, the use of electrically driven tools, which in some cases disturb, degrade or overshadow, partially or totally, the signals of interest. Hence, manual stimulation has often been used in fMRI experiments, albeit this form of stimulation is often accompanied by considerable variance. For example, in one assessment of the somatotopy of the finger's Brodmann area $3 \mathrm{~b}$ using manual tactile stimulation, an intra-examiner error of $18 \%$ has been reported [19]. Furthermore, high costs, as well as long development times to obtain adequate equipments

\footnotetext{
* Corresponding author. Tel.: +41 4463254 04; fax: +41 446321204.

E-mail address: candia@ collegium.ethz.ch (V. Candia).

1 These authors contributed equally to this work.
}

contribute to the limited availability of technical solutions. To better interpret brain dynamics, accurate control of stimulus conditions is mandatory. This is particularly important in the somatosensory system because of the fine-tuned functional properties of the tactile receptors of the human hand [10-12]. One of the main advantages of using electronically driven devices to carry out research studies, is that stimulation protocols can be computer controlled. Brief pneumatic, non-painful sensory stimulation to the skin has been already successfully applied to map the cortical representation of different body parts in healthy subjects and patients using magnetoencephalography $[3,4,16]$. In addition, in fMRI, pneumatic stimulation has been used in different research protocols but has not been incorporated as a standard tool in clinical or research settings so far. In this line of attempts, the somatotopy of the human arm has been investigated by using a series of air puffs along the lower arm [15]. Furthermore, vibrotactile pneumatic stimulation has been used as stimulus for the palm of the hand 
[7] and for finger-tips [5]. Because different stimuli have been used across laboratories, potentially different impacts on brain dynamics can be expected. Replication of stimulus type and its associated effects on BOLD signals appears necessary to allow for meaningful comparisons between studies. While electrical stimulation may stimulate different afferent fibers diffusely [8], it has been shown that the pneumatic stimulation of the fingertips used in the present study activates the human primary sensory cortex or SI [2,3]. Only a few successful attempts to optimize pneumatic stimulation protocols for fMRI, suitable for research and clinical environments have been published. In these experiments a pneumatic system producing non-painful stimuli of $50 \mathrm{~ms}$ length, with a frequency of $4 \mathrm{~Hz}$ and an air pressure of 3 bar applied to finger clips with flexible membranes was used. Several membranes were used to depict the activity resulting from stimulation of the lips, hands and toes $[17,18]$. These data deserve special interest, because they offer one of these few examples of investigation of the somatosensory system using fMRI techniques under controlled conditions. Further implementation of such stimulation paradigms may permit to build bridges between different recording techniques, like MEG, EEG and fMRI; if identical stimulation tools could be used in all these brain-imaging methodologies, results would be comparable in a more conclusive way. Until now, a portable and low-cost pneumatic stimulation system compatible with the fMRI-environment has not been reported. Therefore, we aimed at constructing a device, which has a low-cost and is easy to reproduce. In doing so, we make use of components commercially accessible to a wide range of researchers. A high mobility should be guaranteed to allow its use in different settings, within and between research laboratories and clinical environments, facilitating cooperative work and clinical assessments. To test for the efficacy and accuracy of the system, we induced activity in primary and secondary cortical areas of the somatosensory system. This activity has been consistently reported in other studies, using similar stimulation for the finger pads [13]. Moreover, we wanted to test whether stimulation of a single finger would produce measurable signal changes using fMRI.

All participants gave their written consent before the study, and were fully informed about the procedures and aims of the experiment according to the guidelines of the Helsinki convention for the treatment of experimental subjects. We measured nine volunteers, three females and six males (mean age $31.4 \pm 6.2$ range $26-46$ ). All participants were right handed according to the Edinburgh Handedness Inventory [14]. They were healthy volunteers without any history of neurological disorders as stated in self-report.

The stimulus device was developed at the Department of Psychology of the University of Konstanz, Germany. The controller unit is equipped with two 25 pins SUB-D-interfaces so that it can be connected to the stimulus computer. TTL-logic integrated circuits (74HC245) analyse the incoming signals and generate TTL pulses, which are then amplified from 5 to $24 \mathrm{~V}$ and used to drive a magnetic solenoid valve (525146 MHE3-M1H-3/2G1/8; Operating pressure $-0.9-8$ bar, Festo ${ }^{\circledR}$, Germany). The device permits the operation of up to eight independent chan- nels connected by means of standard cables to the corresponding number of valves. Incoming signals can be visualized by LED's located in the front panel of the device aligned with their corresponding buttons for manual activation. The incoming airflow drives a circular synthetic membrane of approximately $0.8 \mathrm{~cm}^{2}$ (4D Neuroimaging Inc., San Diego, USA) through plastic standard tubes (PUN-4x0,75-SI; outside diameter $4 \mathrm{~mm}$, inside diameter $2.6 \mathrm{~mm}$ and PUN-3x0,5-SI; outside diameter $3 \mathrm{~mm}$, inside diameter $2.1 \mathrm{~mm}$; copper and Teflon-free for QS pushin fittings, Festo ${ }^{\circledR}$ ). The membranes then stimulate the desired skin area whenever the valve is opened. Stimulation was computer driven by means of the commercial software Presentation ${ }^{\circledR}$ (Neurobehavioral Systems, Inc., Albany, CA, USA) running on a standard PC (Fig. 1).

We placed the valve at about $4 \mathrm{~m}$ distance from the MRscanner and attached it to a fixed object within the magnet's room. The minimum distance from the valve to the scanner may vary depending on the MR system's field strength, and has to be considered in order to avoid valve disturbances. To minimize stimulus shape degrading, the length of the tube connecting the stimulation membrane to the valve was reduced to $5 \mathrm{~m}$, but still permitted fully free positioning of the subjects in the bore of the MR-system. Air pressure was delivered using a standard compressed air bottle positioned outside of the magnet room, and was set at $3500 \mathrm{hPa}(=3.5 \mathrm{bar})$. The high-pressure air was regulated in two separate steps to control the amount of air pressure being sent through the tubes to the end membranes. We applied brief pneumatic stimulation to the right thumb. By using plasters, and in order to counteract possible displacements, we fixed the membranes to the mid-volar surface of the distal phalanx of the right thumb, which covered approximately $0.8 \mathrm{~cm}^{2}$ of skin area. Parameters regarding shape and intensity of the stimulus at the delivery site were not tested because the membranes were identical to those routinely used in magnetoenchephalographic measurements under similar conditions. In addition, these membranes are commercially available by 4D Neuroimaging Inc. (http://www.4dneuroimaging.com/).

During measurements, volunteers were instructed to close their eyes. Head movements where mechanically minimized by means of foam rubber cushions. Lights in the MR chamber were dimmed. Functional imaging was performed in a block design with alternating rest and activity conditions, starting with a rest condition. Each block consisted of 7 volumes $(21 \mathrm{~s})$. In total 18 activity blocks were acquired per experiment. We used three different stimulus frequencies of 1,3 and $5 \mathrm{~Hz}$. Each activity block contained one out of these three frequencies (stimulus length $100 \mathrm{~ms}$ ), i.e. each frequency condition was randomly delivered six times during the experiment. The stimulus length used might constitute the upper limit for these membranes. Longer stimulus durations might provoke membrane rupture (with no danger for the subjects) at the stimulation magnitudes we employed (personal experience). All measurements were performed on a $3 \mathrm{~T}$ MR scanner (Philips Achieva) using a six-channel head coil. Functional images were acquired using a single-shot field echo EPI sequence $(\mathrm{TE} / \mathrm{TR}=35 / 3000 \mathrm{~ms}$, FOV $=220 \mathrm{~mm} \times 220 \mathrm{~mm}$, matrix $=128 \times 128$, slices $=39$, slice thickness $=3 \mathrm{~mm}$, slice gap $=0, F A=82$ ) with a SENSE factor 


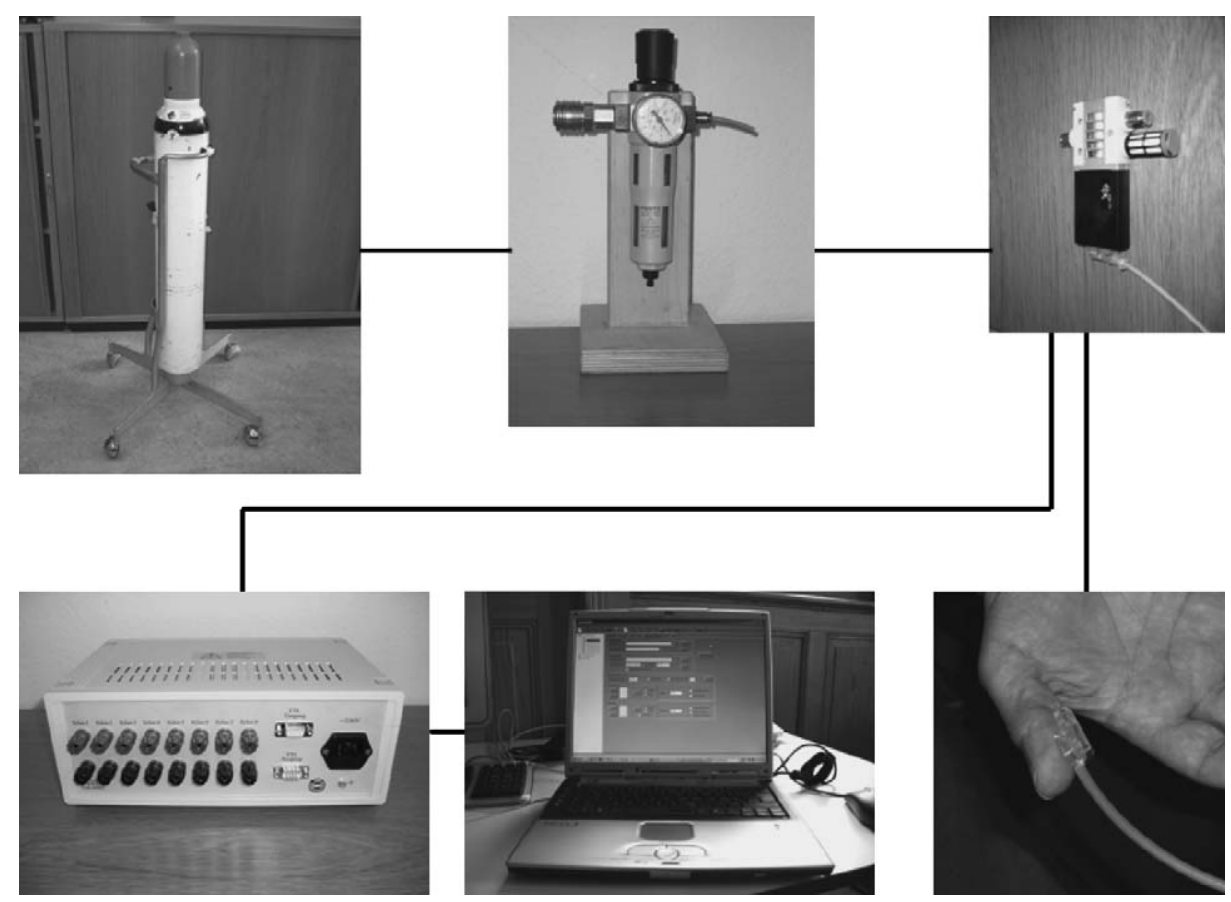

Fig. 1. Stimulus equipment: In the upper panel, the compressed-air bottle (left), the air pressure regulator (middle: real dimension approximately $20 \mathrm{~cm}$ height) and the magnetic solenoid valve (right) are depicted. In the lower panel, a front view of the $20 \mathrm{~cm} \times 29 \mathrm{~cm} \times 11 \mathrm{~cm}$-control unit is shown on the left connected to a standard PC (middle) and to the solenoid valve. The circular synthetic membrane is connected to a plastic standard tube, and to the valve (upper right) and attached to the thumb by means of plasters (lower right). The solid lines represent connections between the constitutive elements of the stimulation system. Note that the air bottle is the only non-portable element in the system.

of 2. Anatomical images were acquired using a 3D T1W field echo sequence $(\mathrm{TE} / \mathrm{TR}=2.3 / 20 \mathrm{~ms}, \mathrm{FOV}=220 \mathrm{~mm} \times 220 \mathrm{~mm}$, matrix $=256 \times 256$, slices $=180$, slice thickness $=0.75 \mathrm{~mm})$. Before its use, the anatomical images were transformed to isovoxel size $(1 \mathrm{~mm} \times 1 \mathrm{~mm} \times 1 \mathrm{~mm})$, and to Tailarach space.

Data analysis was performed with BrainVoyager QX $1.4^{\circledR}$ software package. In the single subject analysis, we used a cluster size of at least 100 voxels as a contiguity threshold for those cases not reaching significance after correction with $q(\mathrm{FDR})<0.05$. For data evaluation, a whole brain strategy was used. To remove unwanted signal components, data pre-processing was done for any subject prior to the computation of group analyses. To diminish arbitrary selection of pre-processing parameters, the standard parameters implemented in BrainVoyager QX 1.4 were adopted. Images were 3D motion corrected by means of trilinear interpolation. We did not correct for slice scan-time because a block design was used. Spatial smoothing was performed with a kernel $=4 \mathrm{~mm}$ voxel size-FWHM, thus, allowing the integration of signals in an area of less than a centimeter. Within this range, smoothing merely reduces the noise by simultaneously enhancing the effects of interest in the signal [6]. Temporal smoothing included the parameters linear trend removal and high pass filter (limited to three cycles). Image sets still contaminated with strong movement artefacts after 3D movement correction were excluded from further analysis [17]. This was done by visually inspecting sudden slope deflections in the graphical representation of the previously performed motion correction in any single data set, and by comparing this with the signal changes in arbi- trarily selected ROI signal time courses of the same subject's data. Scans containing sudden slope deflections in parallel with abrupt signal enhancements were excluded from further analysis as these signals were considered to be "false positives". Before group analysis, all functional and anatomical images were transformed into the Tailarach standard space. In the group analysis, no further data pre-processing (e.g. spatial smoothing) was used. This was done to obtain a reasonably good spatial resolution. Significance level was set at $q(\mathrm{FDR}) \leq 0.05$. To calculate the maximal percentage BOLD signal changes for the group, we first computed a standard General Lineal Model comprising the whole brain using the multi-study option in the analysis software. Thereafter, the signal time courses of any individual subject within the region showing significant activations in the somatosensory cortex, were exported for further external computation. These single contributions were then used to compute a baseline, which was the average of data points corresponding to changes below $5 \%$ of the reference function. This function was modified to account for the haemodynamic delay of the fMRI signal by using the software parameters. Thereafter, the maximal percentage BOLD-signal change of any single subject was computed for each frequency averaging those percent signal changes corresponding to the segment ranging between 95 and $100 \%$ of the reference time function. The percent signal change at point $X$ was defined as: $\%$ signal change $=(($ intensity at point $X$ - baseline)/baseline) $\times 100$. The group maximal percent signal change for a region was the average of all single maximal signal changes for a determined frequency in this region. Singlesubject and group statistical analyses were both computed by 
running a standard General Lineal Model due to the low amount of cases included in our sample. For those brain areas for which the assumption of similar organization among subjects is generally accepted, even short samples allow for generalizations to the whole population [6]. Differences between conditions were assessed with contrasts. Differences in maximal percent signal changes among different cortical regions were calculated using repeated measurement ANOVAS and Fisher tests. Significance level was set to $p<0.05$. Slopes of the BOLD-signal changes were examined generating event related plots within BrainVoyager QX, with an epoch based baseline computation using two data points (e.g. volumes) before stimulus onset.

No image degrading or valve functioning abnormalities due to proximity of the valve and the scanner were observed. Stimuli were all delivered without time disturbances as recorded in the Presentation's event protocol. Air pressure set at $3.5 \mathrm{bar}$ above normal pressure level was high enough to produce membrane stimuli resulting in significant activations in somatosensory areas of the brain cortex at any of the used frequencies of interest.

After correction for multiple comparisons, within subjects frequency comparisons with contrasts were not significant. Therefore, all three frequencies were averaged. Inspection of the single subject activations revealed activation in the postcentral gyrus corresponding to SI in all nine subjects (see Fig. 2 lower panel for some examples). Six subjects showed additional contralateral, and two subjects ipsilateral activation of secondary somatosensory cortex, in the posterior part of the subcentral gyrus (five subjects corrected for multiple comparisons $q(\mathrm{FDR}) \leq 0.05)$. Among subjects, BOLD-signal clusters were confined to similar locations within the hand region of the left post-central gyrus.

One cluster within SI, ranging from the anterior to the posterior bank of the post-central gyrus was seen when all three frequencies together where contrasted with the rest condition. The cluster was located in short distance lateral, anterior and inferior, to the hand knob [20]. As well, a cluster containing activity in SII ranging to the posterior insula, contralateral to the stimulated site was also observed (see Fig. 2 upper panel and Table 1 for Tailarach coordinates, cluster size and maximal $\%$ signal changes). The event related averaging plot cor- responding to these two significantly activated regions revealed conspicuous differences in BOLD-signal slopes (see Fig. 2 middle panel). The two-way repeated measurements ANOVA with the factors Area (SI and SII) and Frequency $(1 \mathrm{~Hz} / 3 \mathrm{~Hz} / 5 \mathrm{~Hz})$ for the BOLD percent maximal signal changes was significant for the main factor Frequency (d.f. $=2,8, F=5.875, p<0.05$ ). Fisher's PLSD post hoc comparisons revealed that maximal percent signals changes in BOLD-response for the $1 \mathrm{~Hz}$ condition were significantly higher than for the 3 and $5 \mathrm{~Hz}$ conditions, respectively $(p<0.05)$. The contrast for the signal produced by $1 \mathrm{~Hz}$ was significantly higher when compared to the $3 \mathrm{~Hz}$ signal $q(\mathrm{FDR}) \leq 0.05$, resulting in 2 activity clusters within the secondary somatosensory cortex (SII contralateral [75 voxels, Center of Gravity in Tailarach coordinates -48 - 21 21]; SII ipsilateral [327 voxels, Center of Gravity in Tailarach coordinates 55 - 28 16] data not shown). No other contrasts were significant. The two-way repeated measurements ANOVA with the factors SideOfArea (SII ipsilateral and SII contralateral) and Frequency $(1 \mathrm{~Hz} / 3 \mathrm{~Hz})$ for the BOLD percent maximal signal changes corresponding to the areas significantly activated in the contrast mentioned above was significant for the main factor SideOfArea (d.f. $=1,8, F=8.589, p<0.05$ ) revealing higher maximal percent signal changes contralateral to the stimulated site. The factor Frequency was also significant revealing higher maximal percent signal changes for the $1 \mathrm{~Hz}$ condition (d.f. $=1,8, F=43.897$, $p<0.05)$. The interaction SideOfArea $\times$ Frequency was not significant.

An easy to construct, low-cost and portable device compatible with MRI and other research environments was successfully developed. The performed measurements demonstrated that the device is able to work free of disturbances within a $3 \mathrm{~T}$ environment. The functional data replicate patterns and locations of activations within the cerebral cortex corresponding to those areas commonly reported after tactile stimulation of the fingers. In addition, the data demonstrated that it is possible to induce reliable and repeatable activity patterns using brief stimuli applied to a single finger with one membrane of the mentioned type, making a comparison between BOLD activities of single fingers feasible for future studies. Previously, reports by Stippich et al. $[17,18]$ demonstrated activation of the contralateral primary somatosensory cortex by means of similar pneumatic stimu-

Table 1

Activated brain areas resulting from the three stimulation frequencies used

\begin{tabular}{|c|c|c|c|c|c|c|}
\hline \multirow[t]{2}{*}{ Frequency $(\mathrm{Hz})$} & \multirow[t]{2}{*}{ Cortical region } & \multicolumn{3}{|c|}{$\begin{array}{l}\text { Center of gravity of clusters in } \\
\text { Tailarach coordinates }\end{array}$} & \multirow[t]{2}{*}{ Number of voxels in cluster } & \multirow[t]{2}{*}{ Maximal percent BOLD signal change } \\
\hline & & $X$ & $Y$ & $Z$ & & \\
\hline \multirow[t]{4}{*}{1} & SIc. & -49 & -23 & 40 & 1302 & 0.43 \\
\hline & SIIc. & -45 & -25 & 18 & 3919 & 0.36 \\
\hline & SIIi. & 52 & -28 & 17 & 325 & 0.30 \\
\hline & SIIi. & 37 & -34 & 15 & 528 & 0.31 \\
\hline \multirow[t]{2}{*}{3} & SIc. & -48 & -22 & 43 & 717 & 0.30 \\
\hline & SIIc. & -41 & -26 & 18 & 1087 & 0.18 \\
\hline \multirow[t]{2}{*}{5} & SIc. & -47 & -22 & 42 & 556 & 0.29 \\
\hline & SIIc. & -45 & -23 & 18 & 555 & 0.12 \\
\hline
\end{tabular}

Contralateral (c)/ipsilateral (i) to the stimulated side. 


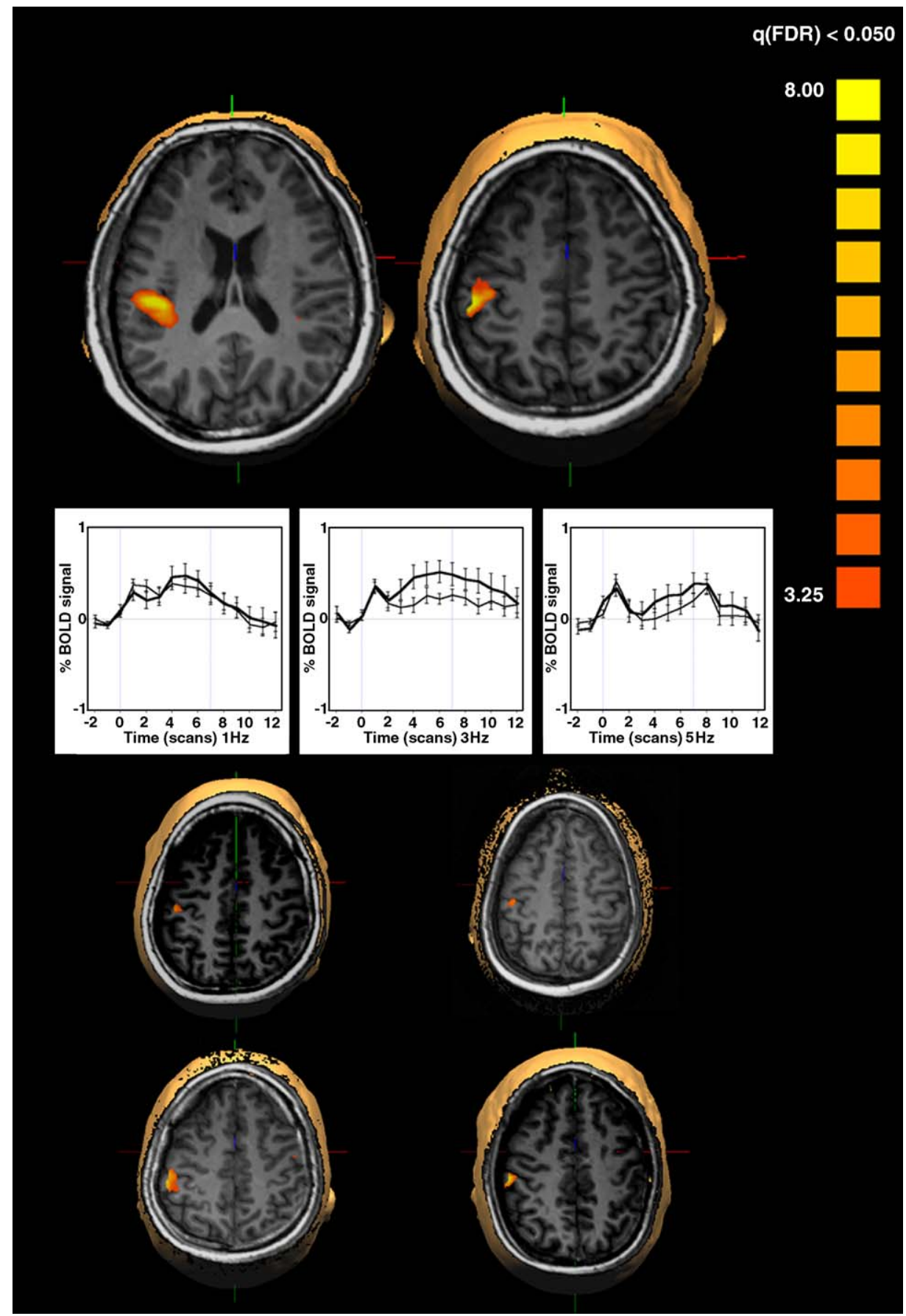

Fig. 2. Upper panel: Activation clusters in the left primary (right) and left secondary (left) somatosensory cortex following pneumatic stimulation of the right thumb (left is left). BOLD-responses for the stimulus frequencies 1,3 and $5 \mathrm{~Hz}$ together are shown superimposed on the anatomical image of one volunteer in the standard Tailarach space (see Table 1 for pertaining information). Middle panel: Event related plots with standard error bars for the $1 \mathrm{~Hz}$ (left), $3 \mathrm{~Hz}$ (middle) and $5 \mathrm{~Hz}$ stimuli (right) within the regions depicted in the upper panel of the figure. Bold lines correspond to primary, thin lines to secondary somatosensory cortex. Low panel: Activation pattern in four single subjects. Shown is the activation on the left post-central gyrus after stimulation of the right thumb. All images were Tailarach transformed. Activity maps are corrected for multiple comparisons $q(\mathrm{FDR})<0.05$. For simplicity, intensity scales for the single subjects have been omitted.

lation. Nevertheless, while Stippich et al. [17] stimulated two fingers simultaneously (D1 and D2), we were able to obtain measurable activations in similar brain areas just by using one membrane. The differences observed among frequencies may be indicative of habituation, to the incomplete recovery of the membranes or to both of them: lower signal changes, as well as unstable BOLD-signal slopes arose at higher frequencies.
Increasing the total amount of skin area being stimulated may also compensate for these differences. This issue needs further investigation. Our results resemble those previously presented by others. This confirms the effectivity of the stimulus being used. Our approach is of relevance not for the study of brain functions in normal volunteers and also for the study of functional changes in the brain of, say the fingers and the lips in such 
neurological pathologies like focal hand and lips dystonia for which distortions of cortical representation have been associated with the sensorimotor disorder, and restoration of dysfunctional cortical representation with illness amelioration [1,2,9]. Further potential uses are the assessment of cortical functionality after reconstruction surgery of the hand, but also the assessment of function recovery after stroke. Other important advantages of this stimulation tool are that there is no need for subjects' cooperation during measurements, stimulation is painless, and the full stimulus device can be set up in approximately $15 \mathrm{~min}$.

The idea of pneumatic stimulation is not new, nevertheless, the development of an easy-to-construct, low-cost and portable device suitable for research and clinical environments might open new possibilities for a wider range of investigators and clinicians interested to study the function of the somatosensory system. The development of non-invasive brain imaging techniques has placed modern scientists in a privileged position in their attempts to study the organization of the living human brain. In particular the somatosensory system has attracted the attention of a whole generation of researchers. Therefore, devices like ours can contribute to a better comparison of results obtained by different techniques like EEG, MEG and fMRI further helping to understand brain functions in the somatosensory system.

\section{Acknowledgements}

This research was supported by the Swiss NCCR on Neural Plasticity and the cogitofoundation. We thank Ramis Movassagh from Collegium Helveticum for valuable comments during the preparation of the manuscript.

\section{References}

[1] S. Butterworth, S. Francis, E. Kelly, F. McGlone, R. Bowtell, G.V. Sawle, Abnormal cortical sensory activation in dystonia: an fMRI study, Mov. Disord. 18 (2003) 673-682.

[2] V. Candia, C. Wienbruch, T. Elbert, B. Rockstroh, W. Ray, Effective behavioral treatment of focal hand dystonia in musicians alters somatosensory cortical organization, Proc. Natl. Acad. Sci. U.S.A. 100 (2003) 7942-7946

[3] T. Elbert, C. Pantev, C. Wienbruch, B. Rockstroh, E. Taub, Increased cortical representation of the fingers of the left hand in string players, Science 270 (1995) 305-307.

[4] H. Flor, T. Elbert, S. Knecht, C. Wienbruch, C. Pantev, N. Birbaumer, W. Larbig, E. Taub, Phantom-limb pain as a perceptual correlate of cor- tical reorganization following arm amputation, Nature 375 (1995) 482484

[5] S.T. Francis, E.F. Kelly, R. Bowtell, W.J. Dunseath, S.E. Folger, F McGlone, fMRI of the responses to vibratory stimulation of digit tips, Neuroimage 11 (2000) 188-202.

[6] R. Goebel, N. Kriegeskorte, Funktionelle magnetresonanztomographie, in: W. Henrik (Ed.), Funktionelle Bildgebung in Psychiatrie und Psychotherapie, Methodische Grundlagen und klinische Anwendung, Schattauer, Stuttgart, New York, 2005, pp. 22-58.

[7] S.M. Golaszewski, F. Zschiegner, C.M. Siedentopf, J. Unterrainer, R.A. Sweeney, W. Eisner, S. Lechner-Steinleitner, F.M. Mottaghy, S. Felber, A new pneumatic vibrator for functional magnetic resonance imaging of the human sensorimotor cortex, Neurosci. Lett. 324 (2002) 125-128.

[8] I. Hashimoto, A. Suzuki, T. Kimura, Y. Iguchi, M. Tanosaki, R. Takino, Y. Haruta, M. Taira, Is there training-dependent reorganization of digit representations in area $3 \mathrm{~b}$ of string players? Clin. Neurophysiol. 115 (2004) 435-447.

[9] Y. Hirata, M. Schulz, E. Altenmuller, T. Elbert, C. Pantev, Sensory mapping of lip representation in brass musicians with embouchure dystonia, Neuroreport 15 (2004) 815-818.

[10] R.S. Johansson, Tactile sensibility in the human hand: receptive field characteristics of mechanoreceptive units in the glabrous skin area, J. Physiol. 281 (1978) 101-125.

[11] R.S. Johansson, A.B. Vallbo, Detection of tactile stimuli. Thresholds of afferent units related to psychophysical thresholds in the human hand, J. Physiol. 297 (1979) 405-422.

[12] R.S. Johansson, A.B. Vallbo, Tactile sensibility in the human hand relative and absolute densities of four types of mechanoreceptive units in glabrous skin, J. Physiol. 286 (1979) 283-300.

[13] J.H. Kaas, R.J. Nelson, M. Sur, C.S. Lin, M.M. Merzenich, Multiple representations of the body within the primary somatosensory cortex of primates, Science 204 (1979) 521-523.

[14] R.C. Oldfield, The assessment and analysis of handedness: the Edinburgh inventory, Neuropsychologia 9 (1971) 97-113.

[15] P. Servos, J. Zacks, D.E. Rumelhart, G.H. Glover, Somatotopy of the human arm using fMRI, Neuroreport 9 (1998) 605-609.

[16] A. Sterr, M.M. Muller, T. Elbert, B. Rockstroh, C. Pantev, E. Taub, Changed perceptions in Braille readers, Nature 391 (1998) 134-135.

[17] C. Stippich, R. Hofmann, D. Kapfer, E. Hempel, S. Heiland, O. Jansen, K. Sartor, Somatotopic mapping of the human primary somatosensory cortex by fully automated tactile stimulation using functional magnetic resonance imaging, Neurosci. Lett. 277 (1999) 25-28.

[18] C. Stippich, A. Romanowski, E. Nennig, B. Kress, S. Hahnel, K. Sartor, Fully automated localization of the human primary somatosensory cortex in one minute by functional magnetic resonance imaging, Neurosci. Lett 364 (2004) 90-93.

[19] D. van Westen, P. Fransson, J. Olsrud, B. Rosen, G. Lundborg, E.M Larsson, Fingersomatotopy in area 3b: an fMRI-study, BMC Neurosci. 5 (2004) 28.

[20] T.A. Yousry, U.D. Schmid, H. Alkadhi, D. Schmidt, A. Peraud, A. Buettner, P. Winkler, Localization of the motor hand area to a knob on the precentral gyrus. A new landmark, Brain 120 (Pt. 1) (1997) 141-157. 